\title{
Comparing osteogenic effects between concentrated growth factors and the acellular dermal matrix
}

\section{Luo HUANG ${ }^{(a)}$ \\ Rui ZOU(a) \\ Jinquan $\mathrm{HE}^{(\mathrm{a})}$ \\ Kexiong OUYANG(a) \\ Zhengguo $\mathrm{PIAO}^{(a)}$}

(a) Guangzhou Medical University, Stomalogical Hospital, Department of Oral and Maxillofacial Surgery, Guangzhou, China.

Declaration of Interest: The authors certify that they have no commercial or associative interest that represents a conflict of interest in connection with the manuscript.

Corresponding Author:

Zhengguo Piao

E-mail:april1007@126.com

htrps://doi.org/10.1590/1807-3107bor-2018.vol32.0029

Submitted: September 03, 2017

Accepted for publication: February 20, 2018

Last revision: March 07, 2018
Abstract: Concentrated growth factor (CGF) is an autogenuous product that contains highly concentrated number of platelets and can be derived from venous blood by selective centrifugation. It has been speculated that local growth factors in human platelets (insulinlike growth factor, IGF; transforming growth factor, TGF-b; platelet derived growth factor, PDGF) would enhance healing of grafts and also counteract resorption. The osteogensis effect of CGF and acellular dermal matrix (ADM) for alveolar cleft defects was evaluated in this study. Twenty alveolar cleft patients were divided randomly into two groups. One group underwent guided bone regeneration (GBR) using acellular dermal matrix film combined with alveolar bone grafting using iliac crest bone grafts (GBR group), while the other group underwent alveolar bone grafting combined with CGF (CGF group). Cone beam computed tomography (CBCT) images were obtained at 1 week and 6 months following the procedure. Using Mimics 17.0 software, the bone resorption rate and bone density improvement rate were calculated and compared between the two groups. Although not significant between ADM and CGF in bone resorption rate, the bone density improvement in cases with CGF(61.62 $\pm 4.728 \%)$ was much better than in cases with $\operatorname{ADM}(27.05 \pm 5.607 \%)(p=0.0002)$. Thus, CGF could be recommended to patients with alveolar cleft as a better choice.

Keywords: Acellular Dermis; Bone Regeneration; Alveolar Bone Grafting

\section{Introduction}

Alveolar bone grafting (ABG) in patients with cleft lip and palate has become an essential part of the surgical management of cleft lip and palate(CLP) patients. The key to the success of this operation is to effectively secure the bone grafts and prevent soft tissue and bacteria invasion into the bone regeneration zone, thus reducing complications such as bone resorption, infection, and dehiscence. ${ }^{1}$ Various methods have been proposed to improve the process of ABG. Currently, the state of the art in-growth factor-aided tissue with regard to reconstruction have been proposed to be an adjuvant of ABG, which can enhance bone formation and specifically to promote bone healing. Additionally, there 
is also a great interest in the $A B G$ procedures that involve use of guided bone regeneration (GBR) to block the invasion of the surrounding soft tissue, allowing sufficient time for osteoblast proliferation in this reserved bone growth space for new bone generation. $^{2}$

Platelet aggregates, such as platelet-rich plasma(PRP) and platelet-rich fibrin (PRF), have been used to accelerate new bone formation associated with GBR for many years. In 2006, Sacco ${ }^{3}$ introduced concentrated growth factors (CGFs), which contain more growth factors and a more rigid fibrin structure; CGFs have recently been used in the dental field. Because platelets are concentrated by varying the centrifuge speed, CGFs are known to contain more rigid and more growth factors than those of previous preparations ${ }^{[3]}$.Therefore, CGFs have been reported recently to be more effective in bone formation or soft tissue healing. ${ }^{4}$

GBR uses acellular dermal matrix (ADM) membranes to block the invasion of the surrounding soft tissue, allowing sufficient time for osteoblast proliferation in this reserved bone growth space for new bone generation. ${ }^{2}$ Clavijo-Alvarez et al. found that using ADM in ABG could prevent postoperative cancellous bone graft exposure without increasing the risk of mucosal disruption or the healing completion time ${ }^{[2]}$. It has been shown that ADM, as a cell membrane bracket, can promote cell adhesion and growth, providing a matrix that is conducive to wound healing. ${ }^{5}$

To our knowledge, studies on the effect of CGF applied in ABG were not reported. Therefore, this study was planned with an aim to compare the osteogenesis efficacy of ADM and CGF for ABG procedure by radiographic analyses with expanded data and to find out clinical recommendation for surgery. The research protocol was approved by the Ethics Committee of Stomatological Hospital of Guangzhou Medical University.

\section{Methodology}

20 patients with alveolar cleft, in the age group of 8-25 years, with unilateral or bilateral cleft lip and palate, who received operation at Department of Oral and Maxillofacial Surgery, Stomatological Hospital of Guangzhou Medical University, from September 2015 to October 2016, were selected. The patients were randomly divided into two groups, the group A (11 patients) received cancellous bone graft from the anterior iliac crest with CGF while in the group B (9 patients), ADM membrane was used during alveolar bone grafting. All participants were assessed against defined inclusion and exclusion criteria. The inclusion criteria were a) a confirmed diagnosis of non-syndromic congenital unilateral complete alveolar cleft without systemic or genetic disorders, b) CLP repair surgery and ABG surgery with iliac crest bone grafting, c) absence of a palatal fistula, infection, or bone graft exposure following alveolar bone grafting surgery, d) clear 3D images of the reconstructed alveolar bone grafting areas were available.

Autologous CGF was prepared from freshly drawn venous blood of the patient. In this study, all patients were subjected to withdrawal of $20 \mathrm{ml}$ of whole blood from peripheral vein of the dorsum of foot. Aliquots of the intravenous blood sample was placed into four tubes. The sample tubes were centrifuged with a CGF centrifuge (Medifuge; Silfradent srl, Sofia, Italy) at 3,000 rpm for $12 \mathrm{~min}$. Centrifugation divided the blood into four layers (Figure 1A), and the second layer or buffy coat and the third layer, also called growth factor layer, were made up of the CGF. ${ }^{6}$ Then the CGF was gently squeezed to be a biofilm (Figure 1B).

ABG was performed under general anesthesia using standard surgical methodology. Bone grafts were harvested from the iliac crest. Cancellous bone grafts of approximately $6 \mathrm{~cm}^{3}$ were harvested using an osteotome. After complete subperiosteal dissection of the alveolar cleft, the nasal lining near the anterior nares was repaired, followed by nasal lining repair in the hard palate. Watertightness of the nasal lining repair was confirmed by flushing normal saline through the nostril. The ADM heterograft (Heal-All; Zhenghai Biotechnology Co., Ltd, Yantai, China) was rehydrated in normal saline for $5 \mathrm{~min}$. Next, a ADM or CGF was placed on the oral side of the nasal lining near the anterior nares and in the hard palate as an onlay graft, 

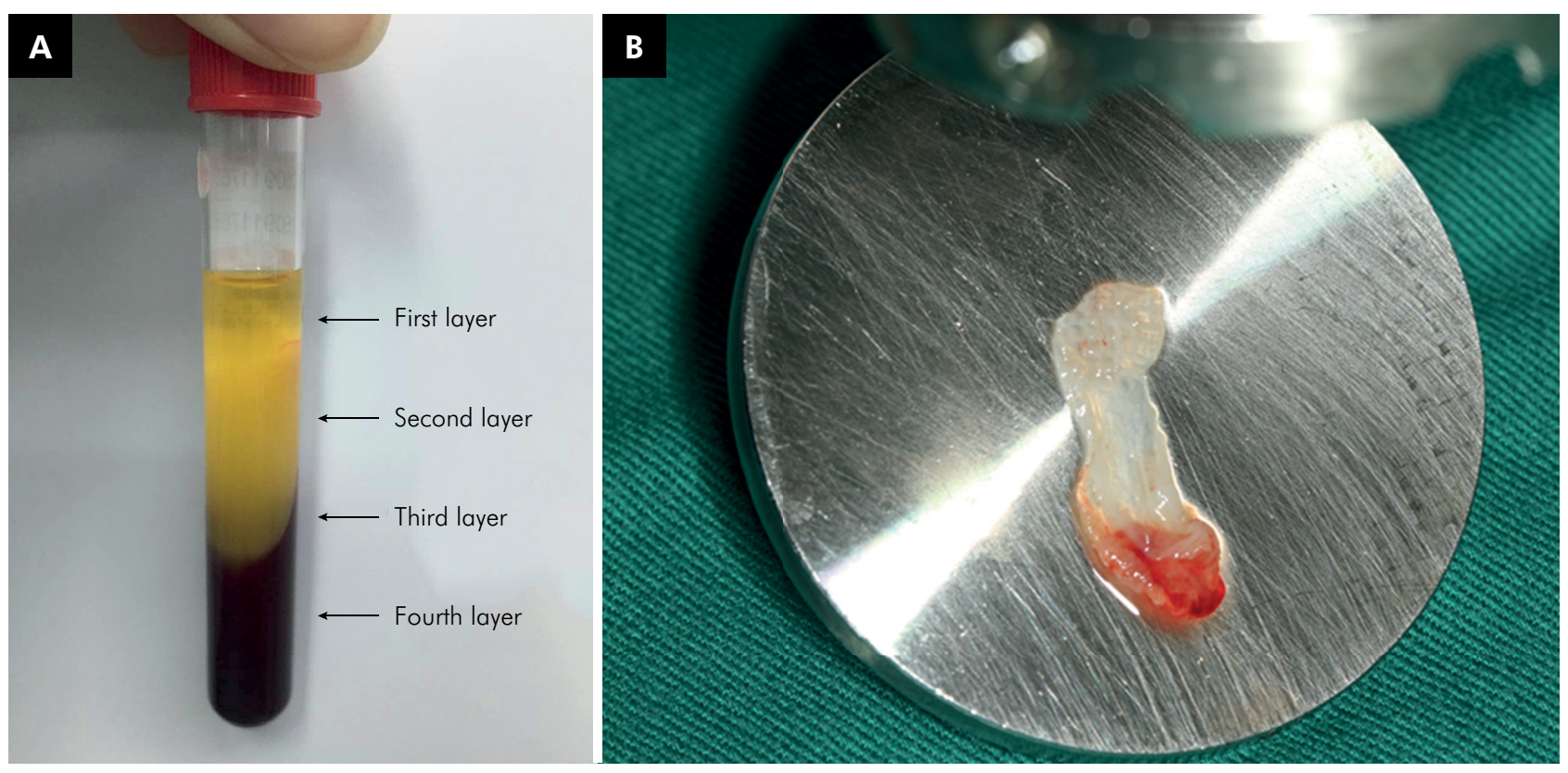

Figure 1. (A). CGF after centrifugation of blood; (B). CGF biofilm

reinforcing the soft tissue repair. The cancellous bone graft, harvested from the iliac crest, was then packed tightly within the bony cleft. Another ADM or CGF was placed over the cancellous bone graft. Finally, the oral mucosal repair was completed over the ADM /CGF.

\section{Image acquisition and analysis}

The CBCT examination was performed at 1 week and 6 months ( \pm 10 days) postoperatively. Data were stored in DICOM format. Images were then uploaded into Mimics 17.0 software (Materialise Inc., Leuven, Belgium) to reconstruct the bone graft area of the alveolar cleft. Measurements were repeated three times on different days with no reference to the original data. The average of the three measurements was used for the final data analysis.

Measurement of the bone graft volume and density as described by Xiao et al ${ }^{[7]}$. The bone graft area was outlined in the coronal, sagittal, and horizontal views. Every plane was labelled layer by layer. The labelled planes were accumulated and imported into Mimics17.0 software 3D images were reconstructed automatically. Finally, the volumes and the bone graft density was calculated.

\section{Statistical analysis}

A paired t-test was used to compare the volume and bone density of the bone graft between CGF group and ADM group at end of 6-month postoperative. All analyses were performed using IBM SPSS Statistics version 22.0 software (IBM Corp., Armonk, NY, USA). A p-value of $\square 0.05$ was considered statistically significant.

\section{Results}

In CGF Group, the average volume of the bone graft was $1995 \pm 771.2 \mathrm{~mm}^{3}$ at 1 week and $1089.38 \pm$ $573.08 \mathrm{~mm}^{3}$ at 6 months after alveolar bone grafting, giving a bone graft resorption rate of $44.47 \pm 4.635 \%$. In the ADM group, the average volume of the bone graft was $1163 \pm 725.6 \mathrm{~mm}^{3}$ at 1 week and $581.74+298.31$ $\mathrm{mm}^{3}$ at 6 months, giving a bone graft resorption rate of $44.31 \pm 5.232 \%$. The bone resorption rate was no significance between the ADM group and the CGF group $(p>0.05)$ (Figure 2) .However, bone grafts with added CGF presented with increased bone density $(61.62 \pm 4.728 \%)$ in comparison to bone grafts with $\operatorname{ADM}(27.05 \pm 5.607 \%)$ at end of 6-month postoperative. A statistically significant improvement in the density was found $(p=0.0002)$ (Figure 3 ). 


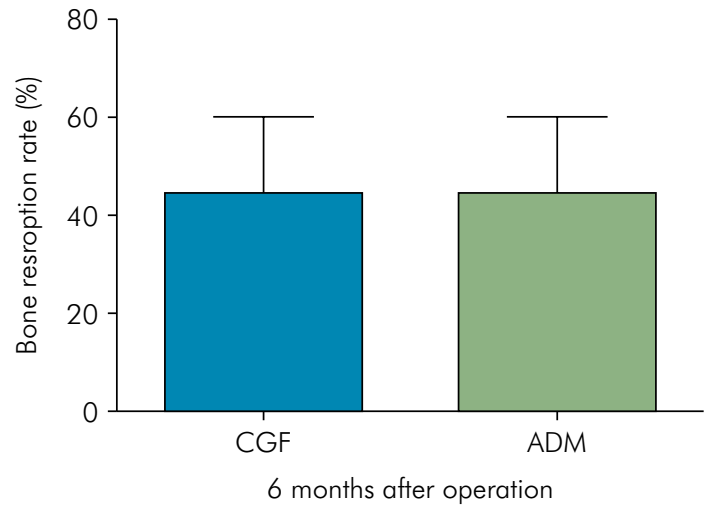

Figure 2. Comparison of the bone density improvement rate between the CGF group and the ADM group at 6 months after alveolar bone grafting $(\mathrm{p}>0.05)$.

\section{Discussion}

$A B G$ is a key step in the treatment of CLP. Boyne and Sands proposed the secondary alveolar bone graft repair theory in $1972 .{ }^{8}$ The accepted time for secondary alveolar cleft repair is between 6 and 12 years. The goal of secondary ABG is not only to repair bone defects, but also to provide the necessary amount of additional bone for prosthetic implant placement and orthodontic treatment. The effect of osteogenesis in the postoperative graft region is a key factor affecting the success of implant surgery and orthodontic treatment.9 Therefore, minimizing bone graft resorption in alveolar cleft patients is pressing issue in ABG research.

At present, GBR is more commonly used in combination with other techniques in clinical practice, especially with bone grafting. The barrier films used in the GBR technique are generally divided into absorbable and non-absorbable membranes. The typical representative non-absorbable membrane is polytetrafluoroethylene (e-PTFE film) and the typical representative absorbable membrane is ADM. But the alveolar bone graft was retained better with the absorbable membrane than the non-absorbable e-PTFE membrane. ${ }^{10}$ The ADM was completely degraded 4-6 months after placement, and the soft tissue and bone tissue became an integral whole. ${ }^{11}$ Additionally, ADM also has a strong resistance to infection and increases gingival keratosis function. ${ }^{12}$ Some studies suggest that the use of ADM membrane in alveolar cleft bone graft surgery can prevent postoperative bone graft exposure

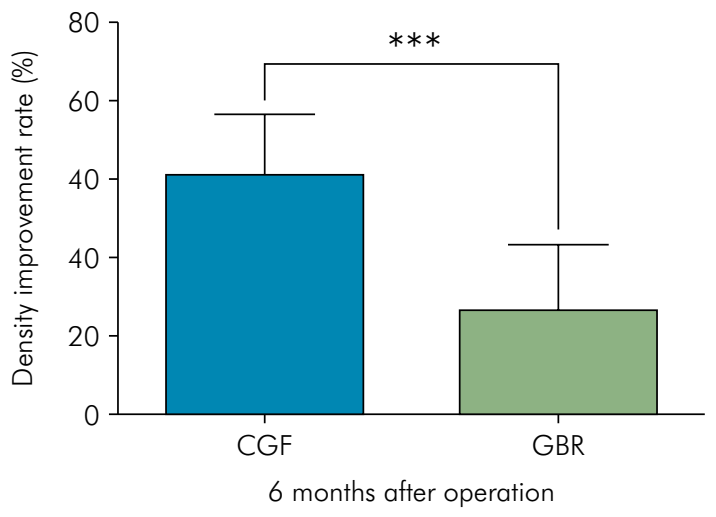

Figure 3. Comparison of the bone density improvement rate between the CGF group and the ADM group at 6 months after alveolar bone grafting ( $p=0.0002)$.

and reduce the risk of infection, and the ADM can be completely replaced by host tissue with no obvious side effects. ${ }^{2,12,13}$ More importantly, the use of ADMmembrane (GBR technique) in alveolar cleft bone grafting would increase the osteogenic effect of the bone graft. ${ }^{7}$

In alveolar bone defect cases, GBR has been shown to be a useful method for treating the bone defect areas. ${ }^{14}$ However, although GBR have been known to show good results, it has shortcomings that create other problems. The shortcomings of GBR are high cost, transmissible diseases and hypersensitivity reactions. To overcome such deficiencies, recently, treatment methods that show good results by grafting platelets obtained from the blood of the patient have been introduced. Among them, PRF showed good results and had thus been used widely in the dental field until recently. ${ }^{14,15}$ However, after the method of concentrating platelets as CGF was introduced, it began to be used as a new material to replace bone grafts. CGFs are simple to make and do not require any synthetics or biomaterials, such as bovine thrombin and calcium chloride, to make gel, so it is free from the risk of cross-contamination. Although CGF has a complex 3-dimensional structure identical to PRF, it had been reported to contain more growth factors than PRF and a more rigid fibrinogen structure. ${ }^{3}$ CGF release growth factors even approximately 7 days after grafting. ${ }^{16}$ Additionally, good results with respect to bone defects have been reported for CGF. ${ }^{16}$ Different to our hypothesis, CGF showed a similar bone resorption 
rate to ADM in our study. This is maybe CGF was applied as a biofilm instead of as a gel block mixed with autogenous bone in our study. Another reason explained for this result is the standard deviation was large and the sample size was small. Although not significant between ADM and CGF in bone resorption rate in alveolar cleft grafting, the bone density improvement in cases with CGF was much better than in cases with ADM. Thus, CGF could be consider as a replacement of ADM .

\section{References}

1. Borba AM, Borges AH, da Silva CS, Brozoski MA, NaclérioHomem MG, Miloro M. Predictors of complication for alveolar cleft bone graft. Br J Oral Maxillofac Surg. 2014 Feb;52(2):174-8. https://doi.org/10.1016/i.bjoms.2013.11.001

2. Clavijo-Alvarez JA, Vecchione L, DeCesare G, Irwin C, Smith DM, Grunwaldt LJ et al. Autologous bone grafting with adjunctive use of acellular dermal matrix for alveolar cleft defects: early outcomes. Cleft Palate Craniofac J. 2010 Mar;47(2):116-21. https://doi.org/10.1597/09-029.1

3. Sacco L apud Park HC, Kim SG, Oh JS, You JS, Kim JS, Lim SC et al. Early bone formation at a femur defect using GF and PRF grafts in adult dogs: a comparative study. Implant dentistry. 2016;25(3):387-93. https://doi.org/10.1097/ID.0000000000000423

4. Dohan Ehrenfest DM, Peppo GM, Doglioli P, Sammartino G. Slow release of growth factors and thrombospondin-1 in Choukroun's platelet-rich fibrin (PRF): a gold standard to achieve for all surgical platelet concentrates technologies. Growth Factors. 2009 Feb;27(1):63-9. https://doi.org/10.1080/08977190802636713

5. Aldekhayel SA, Sinno H, Gilardino MS. Acellular dermal matrix in cleft palate repair: an evidence-based review. Plast Reconstr Surg. 2012 Jul;130(1):177-82. https://doi.org/10.1097/PRS.0b013e318254b2dc

6. Kim TH, Ki, SH, Sándor GK, Kim YD. Comparison of platelet-rich plasma (PRP), platelet-rich fibrin (PRF), and concentrated growth factor (CGF) in rabbit-skull defect healing. Arch Oral Biol. 2014;59(5):5508. https://do.org/10.1016/j.archoralbio.2014.02.004

7. Xiao WL, Zhang DZ, Chen XJ, Yuan C, Xue LF. Osteogenesis effect of guided bone regeneration combined with alveolar cleft grafting: assessment by cone beam computed tomography. Int J Oral Maxillofac Surg. 2016 Jun;45(6):6837. https://doi.org/10.1016/i.ijom.2016.01.013

8. Boyne PJ, Sands NR. Secondary bone grafting of residual alveolar and palatal clefts. J Oral Surg. 1972 Feb;30(2):87-92.

\section{Conclusion}

We conclude that on preliminary investigations, although CGF and ADM have similar bone formation in alveolar clefts when used with autogenous bone chips harvested from the iliac crest, CGF showed higher bone density improvement for alveolar cleft grafting than ADM. Therefore, CGF could be recommended to patients with alveolar cleft as a better choice taking economical and safe factors into consideration.

9. Barbier L, Schepers E. Adaptive bone remodeling around oral implants under axial and nonaxial loading conditions in the dog mandible. Int J Oral Maxillofac Implants. 1997 Mar-Apr;12(2):215-23.

10. Peled M, Aizenbud D, Horwitz J, Machtei EE. Treatment of osseous cleft palate defects: a preliminary evaluation of novel treatment modalities. Cleft Palate Craniofac J. 2005 Jul;42(4):344-8. https://doi.org/10.1597/03-141.1

11. Verschueren DS, Gassner R, Mitchell R, Mooney MP. The effects of guided tissue regeneration (GTR) on modified Le Fort I osteotomy healing in rabbits. Int J Oral Maxillofac Surg. 2005 Sep;34(6):650-5. https://doi.org/10.1016/j.ijom.2005.01.014

12. Helling ER, Dev VR, Garza J, Barone C, Nelluri P, Wang PT. Low fistula rate in palatal clefts closed with the Furlow technique using decellularized dermis. Plast Reconstr Surg. 2006 Jun; 117(7):2361-5. https://doi.org/10.1097/01.prs.0000218788.44591.f0

13. Fowler EB, Breault LG, Rebitski G. Ridge preservation utilizing an acellular dermal allograft and demineralized freeze-dried bone allograft: part II. Immediate endosseous implant placement. J Periodontol. 2000 Aug;71(8):1360-4. https://doi.org/10.1902/jop.2000.71.8.1360

14. Pietrabissa R, Gionso L, Quaglini V, Di Martino E, Simion $M$. An in vitro study on compensation of mismatch of screw versus cement-retained implant supported fixed prostheses. Clin Oral Implants Res. 2000 Oct;11(5):448-57. https://doi.org/10.1034/i.1600-0501.2000.011005448.x

15. Coetzee JC, Pomeroy GC, Watts JD, Barrow C. The use of autologous concentrated growth factors to promote syndesmosis fusion in the Agility total ankle replacement. A preliminary study. Foot Ankle Int. 2005 Oct;26(10):840-6. https://doi.org/10.1177/107110070502601009

16. Sohn DS, Moon JW, Moon YS, Park JS, Jung HS. The use of concentrated growth factors (CGF) for sinus augmentation. J Oral Implantol. 2009;38:25-38. 